\title{
Epitaxial growth of Fe on Mo(110) studied by scanning tunneling microscopy
}

\author{
J. Malzbender, M. Przybylski ${ }^{1}$, J. Giergiel, J. Kirschner * \\ Max-Planck-Institut fur Mikrostrukturphysik, Weinberg 2, D-06120 Halle, Germany
}

Received 20 December 1997; accepted for publication 12 June 1998

\begin{abstract}
Scanning tunneling microscopy has been used to investigate the growth of $\mathrm{Fe}$ on $\mathrm{Mo}(110)$. Growth was carried out at room temperature with growth rates between 0.03 and $0.5 \mathrm{ML} \mathrm{min}^{-1}$. Initially, the first atomic layer is completed independently of the rate of evaporation. At low deposition rates the further growth proceeds in a multi-layer fashion whereas at higher rates the layer growth is partially preserved. Preferred growth along the [001] direction is observed in some cases. One-dimensional dislocation lines are observed in the second-layer islands. At higher coverage a two-dimensional dislocation network occurs which vanishes for thicker films. At a temperature of $600 \mathrm{~K} \mathrm{Fe}$ on $\mathrm{Mo}(110)$ grows by a step flow mechanism in the first layer followed by the formation of wedge-shaped three-dimensional islands. A comparison with Fe grown on W(110) at a similar deposition rate is provided. C) 1998 Elsevier Science B.V. All rights reserved.
\end{abstract}

Keywords: Epitaxy; Nucleation; Scanning tunneling microscopy; Surface diffusion; Surface stress; Wetting

\section{Introduction}

Epitaxially grown ultrathin magnetic films have become subject of considerable attention. The intense research activity in this area is not only driven by the theoretical interest in two-dimensional magnetism, but also by possible applications in magnetic recording and magnetoelectronic devices. Since the magnetic properties can be strongly affected by film continuity and sharpness of the interfaces, the challenge in magnetic studies of ultrathin films is the preparation of well-defined continuous films.

\footnotetext{
* Corresponding author. Fax: (+49) 3455582566.

${ }^{1}$ On leave from: Faculty of Physics and Nuclear Techniques, University of Mining and Metallurgy, al. Mickiewicza 30, 30-059 Krakow, Poland.
}

At thermodynamical equilibrium layer-by-layer growth can be expected if the film wets the substrate. The condition should be fulfilled for each subsequent atomic layer. This will be the case if the heat of adsorption of the adatom onto the substrate is larger than the heat of adsorption onto the adsorbed layer and the surface free energy of the substrate $\gamma_{n-1}$ is larger than the sum of that of the subsequent film $\gamma_{n}$ and its interface with the substrate $\gamma_{n-1} / n$. However, usually film growth does not proceed in thermodynamical equilibrium but is controlled by kinetic parameters. Real surfaces exhibit many defects which provide the additional nucleation centers, such as steps, adatoms adsorbed from the residual gas even under UHV conditions, vacancies and dislocations. Their influence on the nucleation process is provided by the stronger bonding of atoms resulting from the 
increased number of nearest neighbors in adsorption sites and by the disturbances of diffusioncontrolled processes. The thermal energy of the diffusing atoms depends on the substrate temperature. The supersaturation of the condensing atomic beam requires low temperatures or high evaporation rates, whereas a good crystalline order usually requires high temperatures. Therefore, in reality, the film growth is determined by the competition between the influence of defects, substrate temperature and growth rate. In some cases it can result in a layer-by-layer growth even for the nonwetting systems [1].

Another important parameter which determines the structure of epitaxially grown film is the lattice misfit $f$ of the film to the substrate which can be accommodated by elastic strain or by the formation of dislocations $\left(f_{\mathrm{s} / \mathrm{f}}=\left(a_{\mathrm{f}}-a_{\mathrm{s}}\right) / a_{\mathrm{s}}\right.$, where $a_{\mathrm{f}}$ and $a_{\mathrm{s}}$ are the lattice parameter of the film and the substrate respectively). Since the elastic energy of a pseudomorphic monolayer ML is proportional to $f^{2}$, whereas the dislocation energy is proportional to $f$, a critical misfit exists (approximately $10 \%$, depending on the elastic properties of film and substrate) below which pseudomorphic growth can be expected. In addition, the strain energy contributes to the surface energy of the film. As a result, if the strain energy becomes large it could be energetically more favorable to form threedimensional islands on top of the initially continuous film.

Bcc-metal-metal systems, like Fe on W(110), have received considerable interest owing to the strong uniaxial in-plane magnetic anisotropy existing generally in the [110] interface of Fe. The morphologies of Fe films on W(110), as well as their magnetic properties, are well characterized $[2,3]$. In particular, at any temperature $\mathrm{Fe}$ on $\mathrm{W}(110)$ starts to grow with nearly perfect completion of the first atomic layer which is pseudomorphic to the substrate (surface free energy of $\mathrm{Fe}_{\gamma \mathrm{Fe}}=2.55 \mathrm{~J} \mathrm{~m}^{-2}$, surface free energy of $\left.\mathrm{W}_{\gamma \mathrm{W}}=3.3 \mathrm{~J} \mathrm{~m}^{-2}[4]\right)$. For thicker films the equilibrium configuration results in three-dimensional islands grown on the initially existing monolayer and is achieved at elevated temperatures (Stranski-Krastanov mode of growth). Despite the large lattice mismatch between $\mathrm{W}(110)$ and
$\mathrm{Fe}(110), f_{\mathrm{W} / \mathrm{Fe}}=-9.4 \%$, the first monolayer remains pseudomorphic in the $\mathrm{W} / \mathrm{Fe}(110)$ interface independently of preparation temperature and the total film thickness. At temperatures up to about $900 \mathrm{~K}$ no experimental evidence of reconstruction of the films comprised of $\mathrm{Fe}$ atoms in the first atomic layer on $\mathrm{W}(110)$ has been found. This unusual thermodynamical stability of the pseudomorphic $\mathrm{Fe}$ monolayer deposited on tungsten occurs because the strong hybridization between the Fe-d and W-d bands [5] affects the electronic band structure.

An $\mathrm{Fe}$ monolayer on $\mathrm{Mo}(110)$ is another wetting system. In equilibrium, an $\mathrm{Fe}$ film $\left(\gamma_{\mathrm{Fe}}=2.55 \mathrm{~J} \mathrm{~m}^{-2}[4]\right)$ is expected to grow on Mo initially in a layer-by-layer mode owing to the lower surface free energy in comparison with Mo $\left(\gamma_{\mathrm{Mo}}=2.95 \mathrm{~J} \mathrm{~m}^{-2}\right.$, compared with $\gamma_{\mathrm{W}}=3.3 \mathrm{~J} \mathrm{~m}^{-2}$ [4]) and assuming that the interface energy is small (this is actually the case for most of the metal-metal interfaces). The lattice mismatch $f_{\mathrm{Mo} / \mathrm{Fe}}=-8.7 \%$ is near to the value for $\mathrm{Fe}(110)$ on $\mathrm{W}(110), f_{\mathrm{W} / \mathrm{Fe}}=-9.4 \%$. It is below the critical value and pseudomorphic growth of at least the first atomic layer can be expected. Whereas $\mathrm{W}$ is a $5 \mathrm{~d}$ metal, molybdenum is a $4 \mathrm{~d}$-metal, which might have an influence on the hybridization between the d-bands and thereby on the properties of the combined system. Tikhov and Bauer [6] used LEED, AED and work function changes to analyze the growth of $\mathrm{Fe}$ on $\mathrm{Mo}(110)$ at various temperatures. Pseudo-Frank-van der Merwe growth was found near room temperatures. One of their most interesting results is the complex LEED pattern of the thinnest films (about $2 \mathrm{ML}$ ) which was basically interpreted by stepwise transition from the $\mathrm{Mo}(110)$ to the $\mathrm{Fe}(110)$ atomic density in the framework of the periodic lattice distortion model [7]. Another interpretation suggested that the initial reorganization appears not to be a strained bcc-Fe(110) layer, but rather a strained fcc-Fe(111) layer [6]. Any information concerning the strains and quantitative data on their accommodation mechanism would be of great interest in order to distinguish which interpretation is valid.

The aim of this study is the characterization of growth and morphology of $\mathrm{Fe}$ on $\mathrm{Mo}(110)$ with 
scanning tunneling microscopy extending the information obtained with other techniques by Tikhov and Bauer [6]. The influence of growth rate and substrate temperature is studied in an attempt to analyze the details of film growth and to compare the $\mathrm{Fe} / \mathrm{Mo}(110)$ system with the well-characterized system of $\mathrm{Fe}$ on W(110). At equilibrium, similar to $\mathrm{Fe}$ on $\mathrm{W}(110), \mathrm{Fe}$ on $\mathrm{Mo}(110)$ is expected to be a "Stranski-Krastanov system" with a pseudomorphic layer as the equilibrium configuration for the first monolayer [5]. Particular emphasis is placed on the effect of intrinsic strains on the local atomic arrangement.

\section{Experiment}

The experiments were carried out in an ionpumped UHV chamber equipped with an Omicron variable-temperature scanning tunneling microscope (STM) and an Auger electron spectrometer (AES). The Mo(110) crystal was cleaned by repetitive flashing $(400 \times 10 \mathrm{~s})$ at $1300 \mathrm{~K}$ in $10^{-7} \mathrm{mbar}$ oxygen atmosphere to remove the residual carbon contamination. The flashing under oxygen atmosphere was stopped when the AES showed a carbon contamination of less than $2 \%$ of an atomic layer. The oxygen was removed by flashing the sample at $2200 \mathrm{~K}$ for $10 \mathrm{~s}$, which ensured that the oxygen contamination was less than $2 \%$ of an atomic layer. The flashing at $2200 \mathrm{~K}$ was repeated before and after each experiment.

The sample was mounted on a transferable sample holder with an integrated electron beam heater. Iron was evaporated by electron bombardment of a thoroughly outgassed high-purity iron wire at a rate between 0.03 and $0.5 \mathrm{ML} \mathrm{min}^{-1}$. The deposition amount was controlled by an integrated flux monitor cross-calibrated with coverage data obtained from STM images. All evaporations were performed in situ with the sample located in the STM.

The width of terraces on the clean Mo(110) substrate varied between 10 and $100 \mathrm{~nm}$. All STM images were acquired in the constant current mode at currents of 0.1 to $1 \mathrm{nA}$ and bias voltages between +0.1 and $+0.3 \mathrm{~V}$.

\section{Results and discussion}

Fig. 1 shows STM images of Fe films on Mo(110). The films were grown in situ, at room temperature, with a growth rate of $0.03 \mathrm{ML} \mathrm{min}^{-1}$. The images are obtained for coverages ranging from 0.05 to $2.7 \mathrm{ML}$. All images, except Fig. 1a (showing an area free of steps in the image scale) and Fig. 1f, are taken for approximately the same area of the sample. The characteristic monatomic step morphology visible in all the images is of importance for the further discussion, despite of the lack of reproducibility of the same area for all the films. Slight changes of the scanning area observed in almost all the images are due to drift effects. At a thickness of $0.27 \mathrm{ML}$ (Fig. 1b) the nucleation has already finished and the submonolayer islands continue their growth by lateral expansion. The island density at this stage is $2 \times 10^{14} \mathrm{~cm}^{-2}$. Additionally, there is some tendency to decorate the lower edges of the Mo terraces, as is indicated in Fig. 1b. Such decoration is visible owing to the difference in interlayer spacing between $\mathrm{Fe}$ and Mo. The individual islands coalesce into larger islands which are still of the equilibrium shape. Only at some critical island size (corresponding to a coverage of approximately $0.65 \mathrm{ML}$ ) is the coalescence into equilibrium shapes no longer possible for some kinetic reasons. The second layer starts to grow at a coverage of $0.85 \mathrm{ML}$ and reaches its saturation island density $\left(2 \times 10^{14} \mathrm{~cm}^{-2}\right)$ at approximately $1.5 \mathrm{ML}$. At the same coverage the third layer starts to grow. The fourth layer starts to grow at 2.3 ML with a higher nucleation density than that observed in the first and second layers $\left(3 \times 10^{14} \mathrm{~cm}^{-2}\right)$. No preferential growth direction is observed.

Fig. 2 shows the morphology of Fe films grown with a higher deposition rate of $0.2 \mathrm{ML} \mathrm{min}^{-1}$. The second layer starts to grow at $0.9 \mathrm{ML}$, similar to the case of the lower growth rate. The completion of the first layer is observed at a coverage of 1.15 ML, whereas the same coverage obtained with the lower growth rate still produces incomplete filling.

Growth at $300 \mathrm{~K}$ does not proceed in thermodynamic equilibrium and is kinetically limited. At this temperature the diffusion length is below the 

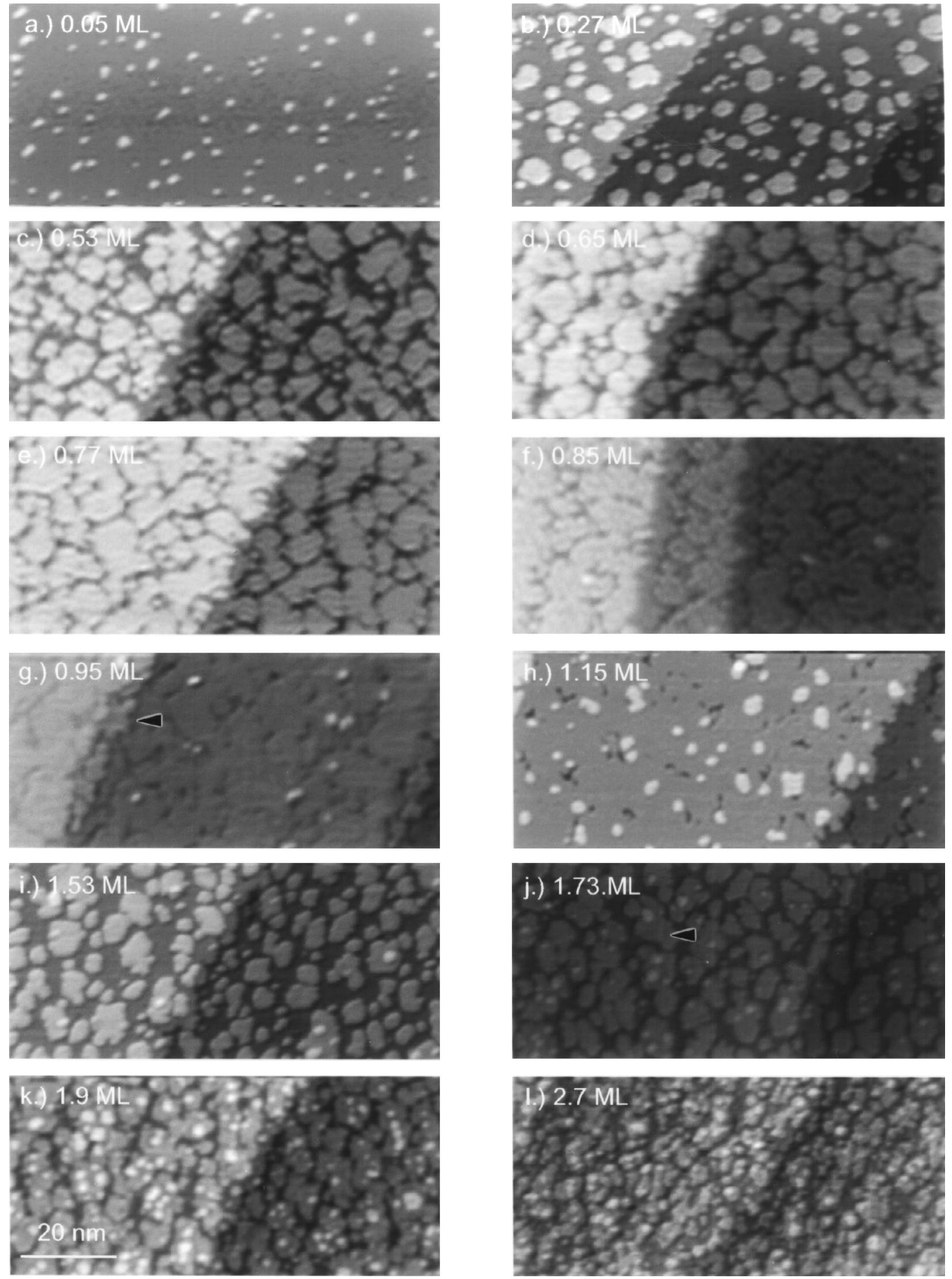

Fig. 1. STM images of $\mathrm{Fe}$ films on $\mathrm{Mo}(110)$. The films were grown in situ, at room temperature, at a growth rate of $0.03 \mathrm{ML} \mathrm{min}^{-1}$. The coverage ranges from 0.05 to $2.7 \mathrm{ML}$. At a coverage of $0.27 \mathrm{ML}$ (b) the nucleation has already finished and the submonolayer islands continue their growth by lateral expansion. Fe decoration of the lower edges of the Mo terraces is indicated in $(\mathrm{g})$. A small dislocation-like feature in the second layer is indicated in the centre of (j) (coverage of $1.73 \mathrm{ML}$ ). No preferential growth direction is observed. 

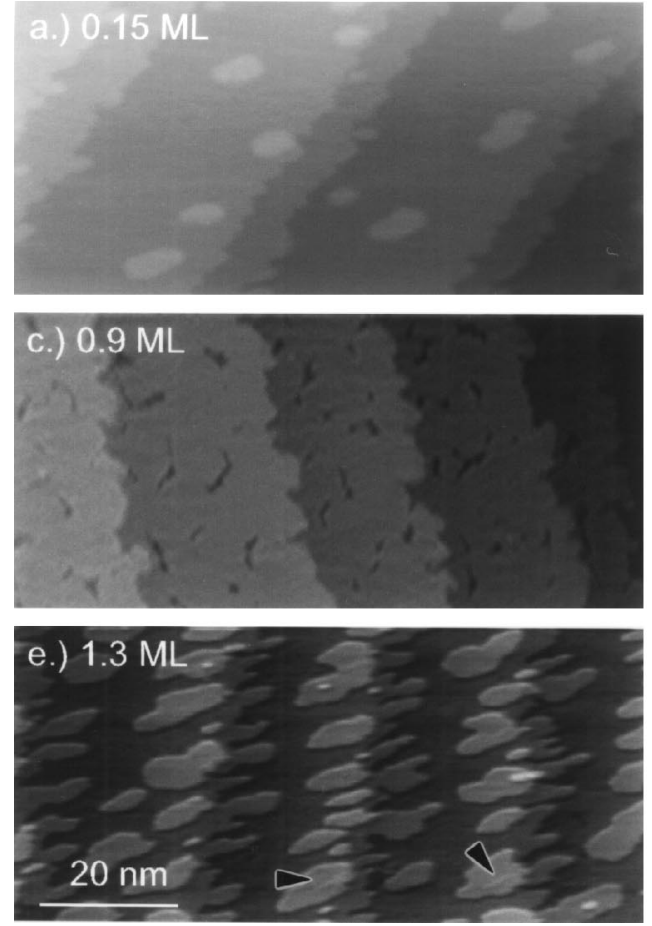
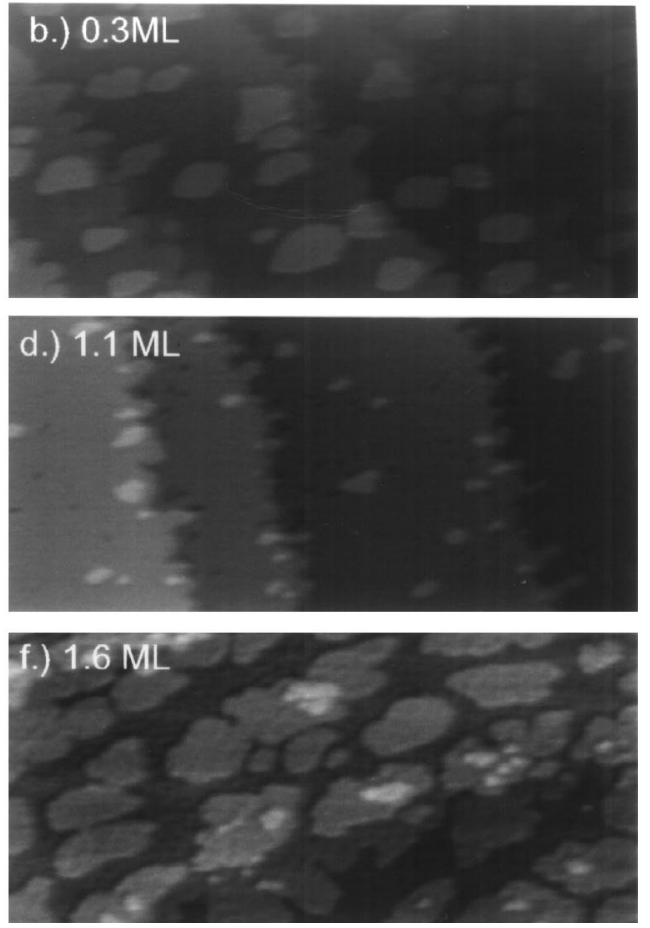

Fig. 2. STM images of Fe films on Mo(110) grown in situ at room temperature with a growth rate of $0.2 \mathrm{ML} \mathrm{min}^{-1}$, i.e. about ten times faster than in Fig. 1. The coverage ranges from 0.15 to $1.6 \mathrm{ML}$. The complete filling of the first layer is observed at a coverage of 1.15 ML. Dislocation features in the second layer are indicated in (e). They are directed along the [001] direction. The secondlayer islands show a preferred growth along the [001] direction and are elongated with an aspect ratio of approximately 1:2. Coalescence is hindered in the second layer, but only along the [110] direction.

average width of the terraces and the film nucleates in two-dimensional islands. Usually, the next layer does not start to grow until the preceding layer is completed. Experimentally, the first layer does, indeed, grow in two dimensions up to its nearly complete filling. The onset of the second layer nucleation is seen at approximately $0.9 \mathrm{ML}$, almost independently of the growth rate.

Fig. 3 compares the STM images for different growth rates at a nominal coverage of approximately $2 \mathrm{ML}(2.2 \pm 0.1 \mathrm{ML})$. With increasing deposition rate the films grow more layer-by-layer, i.e. the next layer starts to grow when the previous one is nearly completed. The higher growth rate also leads to an increase in the size of the second and third layer islands. The size of the second layer islands shown in Fig. 3c, however, may be influenced by the higher step density in this case $(20 \mathrm{~nm}$ terrace width) compared with Fig. $3 \mathrm{~b}$
(40 $\mathrm{nm}$ terrace width). Up to the coverage of approximately $2 \mathrm{ML}$ the growth is only marginally influenced by varying the evaporation rate. However, at higher evaporation rates $(0.2-$ $0.5 \mathrm{ML} \min ^{-1}$ ), the third layer starts to grow at the higher coverage compared with the lower growth rate of $0.03 \mathrm{ML} \mathrm{min}^{-1}$. Such a dependency of the growth fashion on the growth rate agrees with the work of Tikhov and Bauer, who found a clear relationship between the evaporation rate and the coverage at which the third layer starts to grow [6]. This observation is in fact similar to that made in the case of forced layer growth [1]. Accordingly, layer growth can be induced by either a low substrate temperature or a high evaporation rate, even in a non-wetting system. Only at sufficiently low temperatures is the surface mobility reduced and the equilibrium configuration cannot be reached. For a high evaporation rate most of 

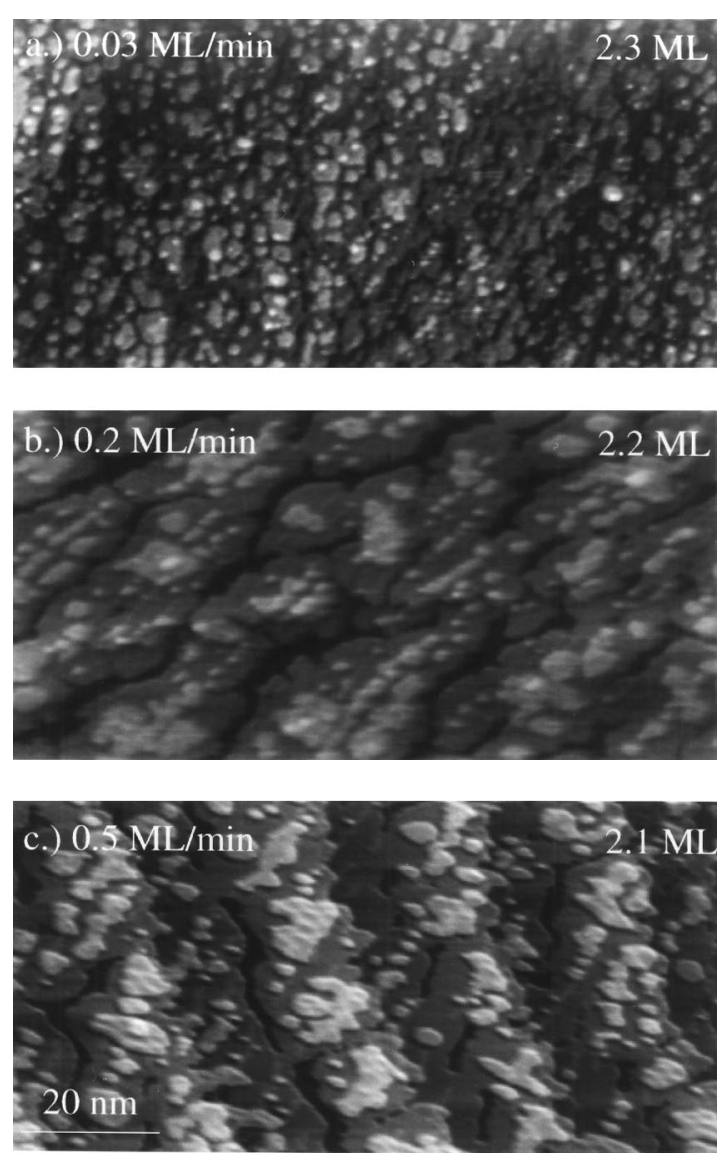

Fig. 3. Comparison of the STM images for different growth rates. Coverage and growth rate are indicated on each individual image. With increasing deposition rate the films grow in a more layer-by-layer fashion, i.e. the next layer starts to grow when the previous one is nearly completed. The higher growth rate also leads to an increased size of the second- and thirdlayer islands. The film grown at a rate of $0.03 \mathrm{ML} \mathrm{min}^{-1}$ (a) shows no dislocation lines at this coverage, whereas the film grown at a rate of $0.2 \mathrm{ML} \mathrm{min}^{-1}$ (b) shows distinct dislocation lines in the [001] direction, which can also be seen at a growth rate of $0.5 \mathrm{ML} \mathrm{min}^{-1}$ (c). Furthermore, the third-layer islands in (c) show indications of a two-dimensional dislocation network of hexagonal shape.

the atoms are adsorbed at the same time, which results in an effect similar to the reduced surface mobility. When the growth rate is too low atoms will diffuse across the surface and result in a larger base of the grown pyramid. Consequently, threedimensional growth can be expected. This corresponds to our observations that the second and further layers grow more in the layer fashion when the growth rate is increased.

There are indications of island coalescence in the first atomic layer at a coverage around $0.65 \mathrm{ML}$. A lack of island coalescence below this coverage, associated with a "dendritic-like" shape of the islands, can be explained by the large lattice mismatch between $\mathrm{Fe}(110)$ and $\mathrm{Mo}(110)$ $\left(f_{\mathrm{Mo} / \mathrm{Fe}}=-8.7 \%\right)$. The resulting stresses can be reduced partly by elastic distortion (bond stretching, bond bending) of $\mathrm{Fe}$ atoms at the island edges [8]. The number of edge atoms is larger if the film consists of a large number of small islands. The coalescence proceeds above $0.65 \mathrm{ML}$, but not in such a narrow coverage range as in the case of $\mathrm{Fe}$ on W(110) [3]. Below completion of the first layer the film should be treated as an adsorbate, and experiments have shown that adsorbates may induce surface stress which is an important stress contribution [9]. The compressive stress (competitive to a tensile stress expected as the Fe film grows pseudomorphically) is suggested to exist, as in the case of Fe on W(110) [2]. This tends to minimize the effective stress down to zero when the first pseudomorphic layer becomes completed and further stabilizes this layer. Therefore, no dislocations are formed in the first atomic layer of $\mathrm{Fe}$ on either $\mathrm{Mo}(110)$ or $\mathrm{W}(110)$. Some role is also played by a strong lateral corrugation of the Fe adsorption potential, which is indicated by the low atom mobility resulting in the high island density observed.

Careful examination of the images shown in the Fig. 1 reveals the presence of small surface features in the second-layer islands. These features have a corrugation character and the visible changes in the image contrast could be of dislocation origin. One of these features is visible in the center of Fig. $1 \mathrm{j}$ (coverage of $1.73 \mathrm{ML}$ ). The main difference between the films grown with high (Fig. 2) and low (Fig. 1) rates is the presence of corrugations in the former ones showing a clear dislocation line character. They can be seen as ridges running across some second-layer islands (Fig. 2e). These ridges correspond to the changes in contrast of the STM image due to the changes in atomic distribution. The misfit dislocations being compensated finally by an inserted extra Fe row is most probably 
expected. They are directed along the [001] direction as determined from the azimuthal orientation of the crystal with respect to the STM scan direction. This relaxation starts at $1.3 \mathrm{ML}$, whereas for the growth rate of $0.03 \mathrm{ML} \mathrm{min}^{-1}$ the relaxation cannot be observed below a thickness of 1.73 ML. The second-layer islands show a preferred growth along the [001] direction and are elongated with an aspect ratio of approximately 1:2. Coalescence is hindered in the second layer, but only along the [110] direction. It is clear that for second-layer islands larger than $80 \mathrm{~nm}^{2}$ (Fig. 1j) the compensation by elastic distortion (which reduces the stress in smaller islands) is no longer sufficient. Apparently, the stress is relaxed by the formation of dislocation lines. This agrees with the observation of the LEED pattern which shows that the thin unannealed Fe film on Mo(110) differs from the thicker film [6], in accordance with the fact that the second and several next layers usually have a packing density intermediate between that of the substrate and that of the bulk film material.

At a coverage of $2.2 \pm 0.1 \mathrm{ML}$ (Fig. 3) the film grown at a rate of $0.03 \mathrm{ML} \mathrm{min}{ }^{-1}$ (Fig. 3a) shows no dislocation lines (islands are too small in the second layer), whereas the film grown at a rate of $0.2 \mathrm{ML} \mathrm{min}^{-1}$ (Fig. 3b) shows distinct dislocation lines in the [001] direction, which can also be seen at a growth rate of $0.5 \mathrm{ML} \mathrm{min}^{-1}$ (Fig. 3c). Furthermore, the third-layer islands in Fig. 3c show indications of a two-dimensional dislocation network of hexagonal shape. This means that, almost independently of the growth rate, the dislocation lines are formed in the second-layer patches, whereas the dislocation networks start to be visible in the third layer. This is again due to the possibility of stress-lowering. Since the lattice expansion is anisotropic, the additional atoms prefer to be located in the crystallographic direction of lower atomic density in comparison with the bulk $\mathrm{Fe}(110)$. This is the reason why the dislocation lines are observed before the dislocation network emerges. In the case of growth rates between 0.2 and $0.5 \mathrm{ML} \mathrm{min}^{-1}$, the preferred growth of the second-layer islands along the [001] direction is observed. This is in fact the same direction as that of the dislocation lines. The atoms inserted in the [110] direction form dislocation lines elongated in the [001] direction. Owing to the more expanded atomic distance in the [110] direction the stress is relaxed in this direction first, in agreement with predictions of the periodic lattice distortion model for a bcc-(110) plane [7]. In general, the atoms prefer the positions of minimum energy, which here is the crystallographic direction that is relaxed due to additional atomic rows.

Growth of $\mathrm{Fe}$ on $\mathrm{Mo}(110)$ at $600 \mathrm{~K}$ (with a growth rate of $0.2 \mathrm{ML} \mathrm{min}^{-1}$ ) is shown in Fig. 4. The growth starting from the edges is observed for those terraces with a width below $30 \mathrm{~nm}$. The submonolayer growth seems to be determined by a step flow mechanism in this case, as can be seen in Fig. 4a. The temperature is high enough and the diffusion length of the atoms is sufficient to reach the edges of steps. Note the clear appearance of fractional steps (indicated in Fig. 4a) which are, as discussed above, due to the electronic effects caused by the difference in interlayer spacing between $\mathrm{Fe}$ and Mo.

At higher coverage the growth changes and most of the material coalesces into large islands. The islands minimize their surface energy by having a flat (110) surface, i.e. the top layer continues across several terrace steps of the underlying Mo substrate and results in a wedge-shaped structure [2]. The aggregation of the clusters is achieved in a similar way as in conventional diffusion-limited aggregation. An adatom is introduced onto a terrace at a large distance from the cluster, but it is sufficiently mobile to undergo a random walk on the terraces. The atoms that reach the terrace edge from above are reflected, whereas the atoms near the lower terrace edge get attracted. The atoms aggregate at a temperature more related to the thermodynamical equilibrium. Three-dimensional islands are built; these increase in size by the material that diffuses close to the edge of the island. The diffusion on the terraces, as well as the diffusion at the terrace edges, is a temperaturedependent process. Since the binding energy at the edges, i.e. the removal of adatoms from island edges, is also an activated process, the size of the two-dimensional $\mathrm{Fe}$ layer on the Mo substrate depends on the temperature of preparation.

Tikhov and Bauer [6] observed two-dimensional growth up to $2.2 \mathrm{ML}$ at $500 \mathrm{~K}$, whereas at higher 

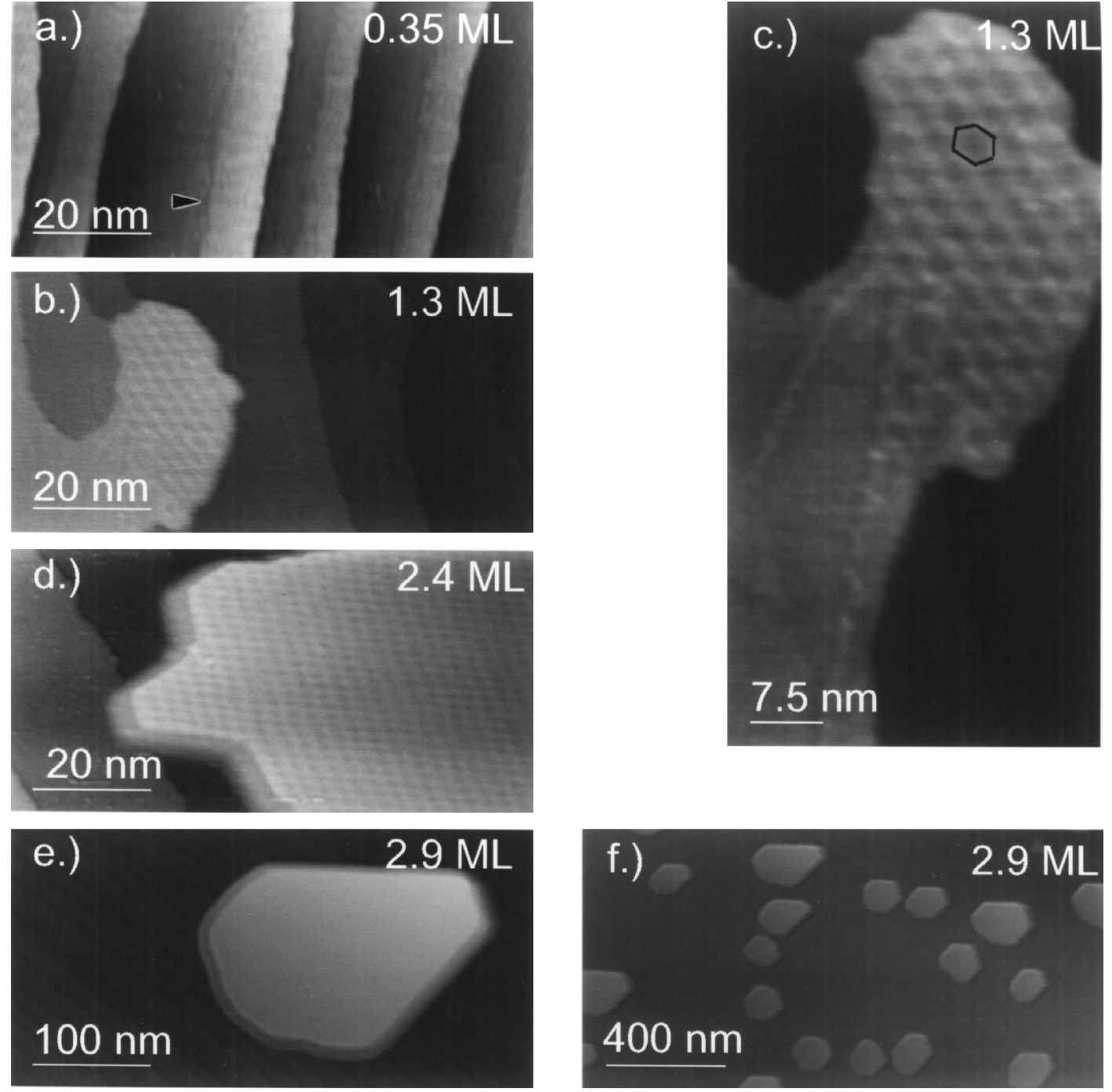

Fig. 4. STM images showing the growth of Fe on Mo(110) at $600 \mathrm{~K}$. The nominal coverage ranges from 0.15 to $2.9 \mathrm{ML}$. At this temperature the submonolayer growth is determined by a step flow mechanism (a). For a total coverage of $1.3 \mathrm{ML}$ (b,c) the dislocations can be seen in the second, third and fourth layers. The two dislocation lines in the second layer are along the [001] direction. They start first as straight [001] lines, but upon approaching the thicker part of the wedge they develop a fine zigzag. In the $3 \mathrm{ML}$ thickness part the dislocation lines merge into a hexagonally shaped dislocation network. One of the hexagons is marked in (c). This dislocation network can easily be observed up to a $7 \mathrm{ML}$ island thickness (d, right-hand side). At higher coverages the Fe coalesces into large characteristically shaped islands as seen in (d-f).

temperature $(660 \mathrm{~K})$ two-dimensional growth exists up to approximately $500 \mathrm{~K}$, since the diffusion on the terrace is enhanced. At higher temperature the three-dimensional islands are formed by the diffusion across the terrace edges. A further increase of the temperature supposedly leads to a diffusion that is independent of the terrace direction, since both the binding energy at the edges and the repulsing barrier at the steps are then negligible compared with the total energy of the particles. This explains observations made by Reuter et al. [10], who found that the diffusion of 
Co on $\mathrm{W}(110)$ at $1073 \mathrm{~K}$ depends on the local morphology of the substrate and that diffusion at $1273 \mathrm{~K}$ is isotropic. The reported anisotropy of the diffusion of $\mathrm{Ni}$ on W(110) even at $1273 \mathrm{~K}$ [10] can be explained by the binding strength at the edges being dependent on the film-substrate combination.

The relaxation process starts in the films prepared at elevated temperatures for a lower coverage in comparison with the films prepared at room temperature. This is due to the formation of the large second-layer patches. Their size is too large to reduce the stresses by elastic distortion at the free edges of islands and, subsequently, dislocations are formed. Owing to larger island sizes and the better atomic ordering the dislocation features are more visible. For a total coverage of 1.3 ML (Fig. 4b,c) they can be seen in the second, third and fourth layers. There is a characteristic difference in dislocation features in Fe layers of different thickness. This is best seen in Fig. 4c. The patch shown here varies in thickness from two (lower left) to four atomic layers (upper right). The two dislocation lines in the second layer are along the [001] direction. They start first as straight [001] lines, but upon approaching the thicker part of the wedge they develop a fine zigzag structure that

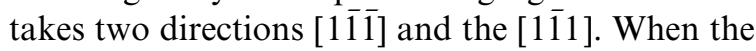
dislocation lines reach the part of the patch that is $3 \mathrm{ML}$ thick the dislocations merge into a hexagonally shaped dislocation network (Fig. 4a). The hexagonal symmetry of this network corresponds to the expected insertion of extra iron rows along the low-density [ $1 \overline{1} \overline{1}]$ and the [1시 1$]$ directions. The dimensions of this hexagonal network are $3.3 \mathrm{~nm}$ and $3.8 \mathrm{~nm}$ in [001] and [1 $\overline{10}$ ] directions respectively. They are similar to those observed for $\mathrm{Fe}$ on W(110) [2] and are in agreement with a compensation of the misfit by insertion of one extra iron row on non-lattice sites every nine atomic rows in both directions in the (110) plane (a lattice mismatch of about $8.7 \%$ means nine atomic distances for the (110) plane of Fe film). The dislocation lines in second-layer islands are formed only in patches of a minimum size of $9 \mathrm{~nm}$. On larger islands they are separated on average by 7 to $10 \mathrm{~nm}$.

When the average coverage is further increased,
Fe coalesces into large characteristically shaped islands as seen in Fig. $4 d-f$. Since the surface is still covered by at least a complete monolayer of $\mathrm{Fe}$, this reflects the change to the Stranski-Krastanov growth. The large islands still form the wedges in the sense of a stepwise varying thickness. In general, the islands show a hexagonal shape (Fig. 4e,f) or a shape resulting from the coalescence of two hexagonal islands. The hexagonal shape of the three-dimensional islands formed at elevated temperatures can be explained if the diffusion process is considered. The atoms that have already reached the clusters are no longer able to leave them, but may hop along the edges. This eventually results in the hexagonal shape of the island [11].

The dislocation network can easily be observed up to a $7 \mathrm{ML}$ thickness of the islands (Fig. 4d, right-hand side). At a thickness of $10 \mathrm{ML}$ (observed for the film of the average coverage of 2.9 ML) they no longer exist in the surface layer of the islands (Fig. 4e,f). However, the surface still reflects the terrace structure of the underlying Mo substrate. Still, an influence of the dislocation network of the hexagonal shape on the morphology of the three-dimensional islands cannot be ruled out, since there are obviously energetically favorable places in the dislocation structure that could influence further growth.

\section{Conclusions}

It can be concluded that the morphology of iron films grown on $\mathrm{Mo}(110)$ at room temperature exhibits a dependence on the growth rate in agreement with the kinetic arguments for the forced layer growth out of equilibrium. The first layer grows in two dimensions up to its nearly perfect completion. In general, the results for $\mathrm{Fe}$ grown on $\mathrm{Mo}(110)$ agree with the results for $\mathrm{Fe}$ on W(110) at similar deposition rates. Since the growth rate was not varied systematically in the experiment of Bethge et al. [2], a dependence of the film morphology on the rate of growth was not known.

At elevated temperatures the StranskiKrastanov mode of growth is found, in agreement 
with the observations of Tikhov and Bauer [6]. For thicker films the coalescence into three-dimensional islands concerns all the material except the first monolayer, which remains continuous and pseudomorphic to the $\operatorname{Mo}(110)$ substrate. The details concerning the formation of three-dimensional islands and their hexagonal shape are explained by kinetically limited processes.

No dislocations are formed in the first atomic layer of $\mathrm{Fe}$ on $\mathrm{Mo}(110)$ owing to the strong $\mathrm{Fe}$ adsorption potential on Mo. The existing stresses are reduced either by bond stretching of $\mathrm{Fe}$ atoms at the island edges or by the compressive stress usually induced by adsorbates. In the second and next atomic layers the stress is relaxed by misfit dislocations; these are visible initially in the form of dislocation lines which form a network of hexagonal shape. All the results concerning dislocation features are interpreted in terms of the periodic lattice distortion model for bcc-Fe(110). No arguments supporting the existence of an $\mathrm{Fe}(111)$ strained layer are found.

The relation between the details of the growth and the resulting magnetic properties needs to be investigated. Films grown at elevated temperatures form three-dimensional islands, which might be of some interest for the development of in-plane recording media.

\section{Acknowledgements}

The technical assistance of Mr. Hunold and Mr. Hartung in the design and construction of the experiment is gratefully acknowledged.

\section{References}

[1] U. Gradmann, Handbook of Magnetic Materials, vol. 7, North-Holland, Amsterdam, 1993, p. 7.

[2] H. Bethge, D. Heuer, C.H. Jensen, K. Reshoeft, U. Koehler, Surf. Sci 331/333 (1995) 878.

[3] M. Przybylski, I. Kaufmann, U. Gradmann, Phys. Rev. B 40 (1989) 8631

[4] L.Z. Mezey, J. Giber, Surf. Sci. 117 (1982) 220.

[5] M. Przybylski, J. Korecki, U. Gradmann, Appl. Phys. A 52 (1991) 33.

[6] M. Tikhov, E. Bauer, Surf. Sci. 232 (1990) 73-91.

[7] U. Gradmann, G. Waller, Surf. Sci. 116 (1982) 539.

[8] J. Massies, N. Gradjean, Phys. Rev. Lett. 71 (1994) 1411.

[9] D. Sander, R. Skomski, C. Schmidthals, A. Enders, J. Kirschner, Phys. Rev. Lett. 77 (1996) 2566.

[10] D. Reuter, G. Gerth, J. Kirschner, in: M.C. Tringidis (Ed.), Surface Diffusion: Atomistic and Collective Processes, Plenum, New York, 1997, p. 489.

[11] M.C. Bartelt, J.W. Evans, Surf. Sci. 314 (1994) L829. 